\title{
Competing Ultrafast Energy Relaxation Pathways in Photoexcited Graphene
}

\author{
S. A. Jensen, ${ }^{\dagger}{ }^{\dagger}$ Z. Mics, ${ }^{\dagger}$ I. Ivanov, ${ }^{\dagger}$ H. S. Varol, ${ }^{\dagger}$ D. Turchinovich, ${ }^{\dagger}$ F. H. L. Koppens, ${ }^{*}$, M. Bonn, $^{*}{ }^{\dagger}$ \\ and K. J. Tielrooij*,§ \\ ${ }^{\dagger}$ Max Planck Institute for Polymer Research, Ackermannweg 10, 55128 Mainz, Germany \\ ${ }^{\ddagger}$ FOM Institute AMOLF, Amsterdam, Science Park 104, 1098 XG Amsterdam, Netherlands \\ ${ }^{\S}$ ICFO - Institut de Céncies Fotóniques, Mediterranean Technology Park, Castelldefels (Barcelona) 08860, Spain
}

\section{Supporting Information}

\begin{abstract}
For most optoelectronic applications of graphene, a thorough understanding of the processes that govern energy relaxation of photoexcited carriers is essential. The ultrafast energy relaxation in graphene occurs through two competing pathways: carrier-carrier scattering, creating an elevated carrier temperature, and optical phonon emission. At present, it is not clear what determines the dominating relaxation pathway. Here we reach a unifying picture of the ultrafast energy relaxation by investigating the terahertz photoconductivity, while varying the Fermi energy, photon energy and fluence over a wide


range. We find that sufficiently low fluence $\left(\lesssim 4 \mu \mathrm{J} / \mathrm{cm}^{2}\right)$ in conjunction with sufficiently high Fermi energy $(\gtrsim 0.1 \mathrm{eV})$ gives rise to energy relaxation that is dominated by carrier-carrier scattering, which leads to efficient carrier heating. Upon increasing the fluence or decreasing the Fermi energy, the carrier heating efficiency decreases, presumably due to energy relaxation that becomes increasingly dominated by phonon emission. Carrier heating through carrier-carrier scattering accounts for the negative photoconductivity for doped graphene observed at terahertz frequencies. We present a simple model that reproduces the data for a wide range of Fermi levels and excitation energies and allows us to qualitatively assess how the branching ratio between the two distinct relaxation pathways depends on excitation fluence and Fermi energy.
\end{abstract}

KEYWORDS: Graphene, ultrafast, hot carrier, terahertz, pump-probe

$G$ raphene is a promising material for, among others, photosensing and photovoltaic applications, ${ }^{1}$ owing to its broadband absorption, ${ }^{2,3}$ its high carrier mobility ${ }^{4,5}$ and the ability to create a photovoltage from heated electrons or holes. ${ }^{6}$ It furthermore uniquely allows for electrical control of the carrier density and polarity. ${ }^{4}$ To establish the potential and limitations of graphene-based optoelectronic devices, a thorough understanding of the ultrafast (subpicosecond) primary energy relaxation dynamics of photoexcited carriers is essential. For undoped graphene (with Fermi energy $E_{\mathrm{F}} \approx 0$ ), ultrafast energy relaxation through interband carrier-carrier scattering was predicted ${ }^{7}$ and observed ${ }^{8,9}$ to lead to multiple electron-hole pair excitation. For doped graphene (with Fermi energy $\left|E_{\mathrm{F}}\right|>0$ ), ultrafast energy relaxation through carriercarrier interaction also plays an important role, with intraband scattering leading to carrier heating. ${ }^{10-13}$ In addition, the ultrafast energy relaxation was ascribed to optical phonon emission, ${ }^{14,15}$ which reduces the carrier heating efficiency. Closely related, for undoped graphene the sign of the terahertz ( $\mathrm{THz}$ ) photoconductivity is positive (see, e.g., refs 16-19), whereas for intrinsically doped graphene the sign is negative, meaning that photoexcitation gives rise to an apparent decrease of conductivity. ${ }^{11,16,17,20-22}$ This negative photoconductivity was attributed to stimulated $\mathrm{THz}$ emission ${ }^{20}$ and to a reduction of the intrinsic conductivity by enhanced scattering with optical phonons $^{21,22}$ or by carrier heating. ${ }^{11,16,17}$ Because the experimental parameters of all these studies differ strongly, different conclusions were drawn regarding the dominating energy relaxation pathway and the origin of the sign of the $\mathrm{THz}$ photoconductivity.

Here, we reach a unifying picture of the energy relaxation of photoexcited carriers in graphene by experimentally studying the ultrafast energy relaxation of photoexcited carriers for a large parameter space, where we vary the Fermi energy and the fluence. We compare the data with a simple model of carrier heating, which quantitatively reproduces the frequency-dependent $\mathrm{THz}$ photoconductivity for a large range of Fermi energies and fluences with a single free fit parameter. This fit parameter is the carrier heating efficiency, that is, the fraction of absorbed energy from incident light that is transferred to the electron system. Although the $\mathrm{THz}$ photoconductivity is not a direct probe of the carrier temperature, and the model parameters

Received: July 18, 2014

Revised: September 19, 2014

Published: September 23, 2014 

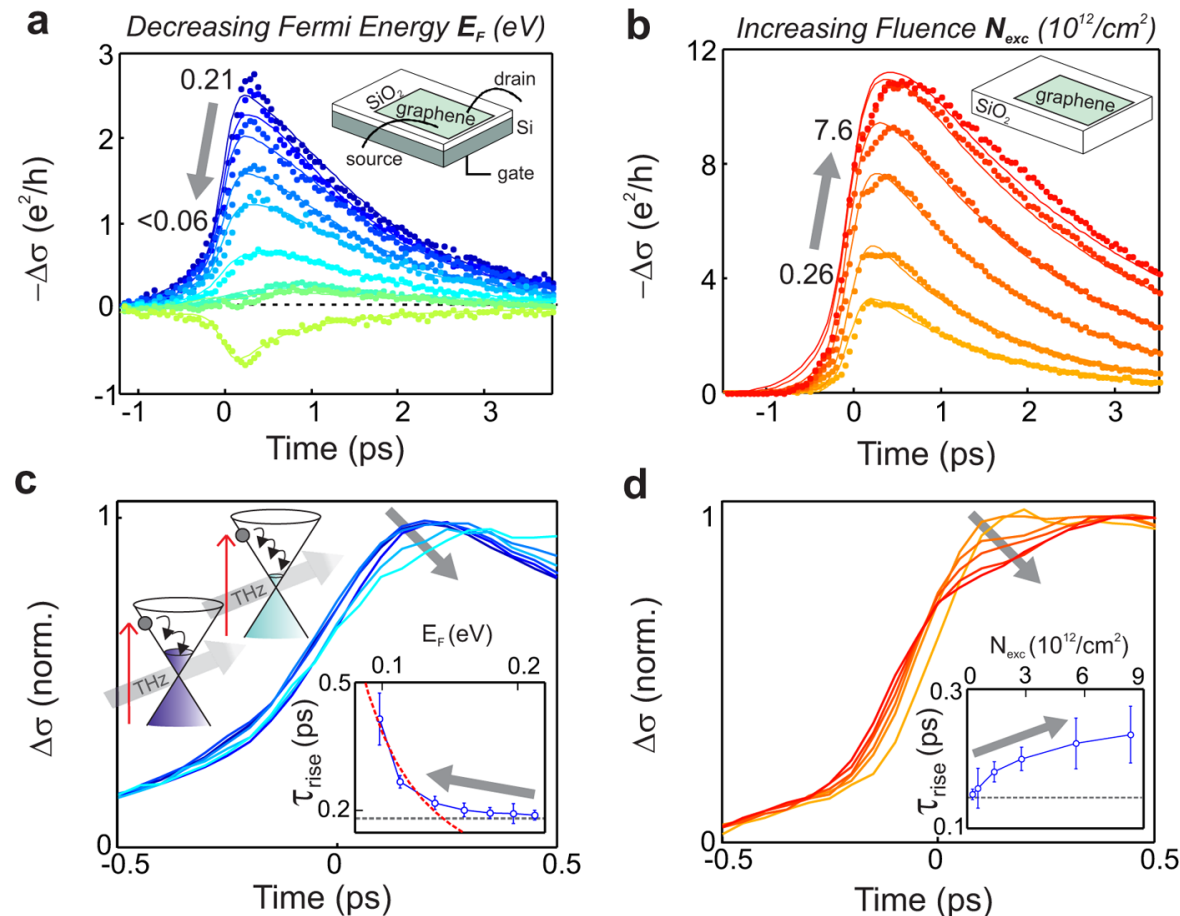

Figure 1. Energy relaxation dynamics-dependence on Fermi energy and fluence. (a) The time-resolved photoconductivity for nine different gate voltages $\left(V-V_{\mathrm{D}}=-70,-60,-50,-40,-30,-20,-15,-10\right.$, and $\left.0 \mathrm{~V}\right)$, that is, for decreasing Fermi energy $E_{\mathrm{F}}$ or intrinsic carrier concentration $N_{\text {int }}$ (sample with controllable Fermi energy). The photoconductivity is extracted from the measured change in THz transmission after photoexcitation with $1500 \mathrm{~nm}$ light (excitation power corresponds to $N_{\mathrm{exc}}=0.8 \times 10^{12}$ absorbed photons $/ \mathrm{cm}^{2}$ ), using the thin film approximation as in ref 11 . The inset shows the geometry of the sample, as explained in the text. The photoconductivity is negative for all traces, except for the one closest to the Dirac point, where it is positive. The solid lines are fits with an effective rise time $\tau_{\text {rise }}$ and an exponential decay time (see Methods). (b) The timeresolved photoconductivity for excitation with $800 \mathrm{~nm}$ light at six different excitation powers, corresponding to absorbed photon densities of $N_{\text {exc }}=$ $0.26,0.50,1.2,2.5,5.0$, and $7.6 \times 10^{12}$ photons $/ \mathrm{cm}^{2}$ (sample with fixed Fermi energy). The geometry of the sample with a fixed Fermi energy of $E_{\mathrm{F}}$ $\sim 0.1 \mathrm{eV}$ (see Supporting Information) is shown in the inset and explained in the text. The photoconductivity is negative and increases for increasing excitation power, as the carrier temperature increases. The solid lines are fits with an effective rise time $\tau_{\text {rise }}$ and an exponential decay time (see Methods). (c) The photoconductivity normalized to the peak of the signal (sample with controllable Fermi energy), showing that decreasing the Fermi energy leads to slower rise dynamics (i.e., slower energy relaxation). The fitted values for $\tau_{\text {rise }}$ as a function of $N_{\text {int }}$ are shown in the inset, together with the theoretically predicted energy relaxation time based on ref 10 (scaled by a factor 1.8) and the experimental time resolution (horizontal dashed line). The schematics in the top left show the ultrafast energy relaxation of a photoexcited carrier for two different Fermi energies, according to the carrier heating process described in refs 10 and 11. (d) The photoconductivity normalized to the peak of the signal (sample with fixed Fermi energy), showing that increasing the excitation power leads to slower rise dynamics. The fitted values for $\tau_{\text {rise }}$ as a function of $N_{\text {exc }}$ are shown in the inset together with the experimental time resolution (horizontal dashed line).

carry some uncertainty, this approach allows us to identify qualitatively how the ultrafast energy relaxation processes depend on excitation fluence and Fermi energy.

We study the ultrafast carrier-energy relaxation using timeresolved $\mathrm{THz}$ spectroscopy experiments, where an excitation pulse with a wavelength of 400,800 , or $1500 \mathrm{~nm}$ (corresponding to a photon energy of $E_{0}=3.10,1.55$, or $0.83 \mathrm{eV}$, respectively) excites electron-hole pairs, and a lowenergy terahertz (THz, $f=0.3-2 \mathrm{THz}$ ) probe pulse interrogates the sample, where it interacts with mobile carriers. An optical delay line controls the relative time delay between excitation and probe pulses, making it possible to determine the photoinduced change in conductivity (the photoconductivity $\Delta \sigma$ ) as a function of time with a time resolution of $\sim 150 \mathrm{fs}^{23}$ We use two different samples: The first sample, where the graphene has a controllable Fermi energy, contains chemical vapor deposition (CVD) grown graphene with an area of $1 \mathrm{~cm}^{2}$ transferred onto a substrate that consists of doped silicon, covered by a $300 \mathrm{~nm}$ thick layer of $\mathrm{SiO}_{2}$. The silicon serves as the backgate for electrical control of the Fermi energy (carrier doping) of the graphene sheet from $E_{\mathrm{F}}=0.3 \mathrm{eV}$ down to $\sim 0.06$ $\mathrm{eV}$ (the width of the neutrality region is $\sim 0.06 \mathrm{eV}$, see
Supporting Information). We use weakly doped silicon (resistivity of $10-20 \Omega \mathrm{cm}$ ) and sufficiently low pump energy $(0.83 \mathrm{eV})$ to ensure that the photoinduced signal from the silicon in the substrate (without graphene) is minimized (see Supporting Information). Two silver-pasted electrical contacts to the graphene sheet enable resistance measurements to retrieve the Fermi energy through the capacitive coupling of the backgate (see Supporting Information). The second sample contains CVD grown graphene with an area of a square inch transferred onto $\mathrm{SiO}_{2}$ with a fixed Fermi level of $<0.15 \mathrm{eV}$ (see Supporting Information). We carefully measure the fluence $F$ as described in ref 24 and use the experimentally determined optical absorption of graphene on $\mathrm{SiO}_{2}$ using standard UV-vis spectrometry to obtain the absorbed fluence $F_{\mathrm{abs}}$, and from this the number of absorbed photons $N_{\mathrm{exc}}=F_{\mathrm{abs}} / E_{0}$, which is equal to the number of primary excited carriers.

To investigate the ultrafast (subpicosecond) energy relaxation dynamics of photoexcited carriers, we first examine the temporal evolution of the photoconductivity. We show in Figure la the dynamics for a range of Fermi energies using the sample with controllable Fermi energy and in Figure $1 \mathrm{~b}$ the dynamics for a range of fluences using the sample with fixed 
Fermi energy. We find that all traces, except the one with Fermi energy within the neutrality region width $\left(E_{\mathrm{F}}<0.06 \mathrm{eV}\right)$, show negative photoconductivity with a subpicosecond rise, followed by a picosecond decay. Similar pump-probe dynamics have been observed before ${ }^{11,16-22}$ and can be understood as follows: During the rise of the signal three main processes take place: (i) the creation of initial electron-hole pairs; and subsequent ultrafast energy relaxation through two competing relaxation channels, namely (ii) carrier-carrier scattering and (iii) optical phonon emission. Thus, the peak signal corresponds to a "hot state" with an elevated carrier temperature $T_{\mathrm{el}}$ and/or more energy in optical phonons. ${ }^{8,10-15}$ During the subsequent picosecond decay, the hot state cools down to the same state as before photoexcitation. The fraction of absorbed energy that after the initial ultrafast energy relaxation ends up in the electron system or in the phonon system depends on the time scales associated with carrier heating and phonon emission, respectively.

The ultrafast energy relaxation takes place during the first few hundred femtoseconds after photoexcitation, that is, during the rise of the conductivity change. Figure $1 \mathrm{c}, \mathrm{d}$ shows the normalized photoconductivity signals for this time window. We will discuss the evolution of the signal amplitude as a function of Fermi energy and fluence later. First we note that the rise dynamics exhibit an intriguing effect: upon decreasing the Fermi energy (i.e., the density of intrinsic carriers $N_{\text {int }}$ ) or increasing the fluence (i.e., the density of primary excited carriers $N_{\text {exc }}$ ) the signal peak is reached at increasingly later times. To quantify these results, we describe the dynamics using two rise times and an exponential decay time. The two rise times allow for part of the conductivity change to occur with the (fixed) experimental time resolution and part with a (free) slower time scale. We then examine the effective rise time $\tau_{\text {rise }}$, which is the amplitude-weighted average of the two (see Methods). The insets of Figure $1 \mathrm{c}, \mathrm{d}$ show $\tau_{\text {rise }}$ as a function of $N_{\text {int }}$ and $N_{\text {exc }}$ respectively. Indeed, for decreasing $N_{\text {int }}$ and increasing $N_{\text {exc }}$ the effective rise time increases from below 200 fs (limited by the experimental time resolution) up to $400 \mathrm{fs}$ (for $E_{\mathrm{F}}<0.1 \mathrm{eV}$ ).

The slowing down of the ultrafast energy relaxation of photoexcited carriers with decreasing Fermi energy is consistent with energy relaxation through intraband carriercarrier scattering (see bottom right inset in Figure 1c). The microscopic picture of this scattering process is shown for two different Fermi energies in the top left inset of Figure 1c. Photoexcited carriers relax by exchanging energy with intrinsic conduction band carriers that thus heat up. The amount of energy that is exchanged between photoexcited carriers and intrinsic conduction band carriers in each intraband carriercarrier scattering event is $\sim E_{\mathrm{F} .}{ }^{10}$ Therefore, if $E_{\mathrm{F}}$ decreases more energy-exchange events are required for the photoexcited carriers to complete their energy relaxation cascade and therefore the relaxation time will increase (see bottom right inset of Figure 1c). If the energy relaxation through carriercarrier scattering would slow down in such a way that the relaxation rate becomes comparable to the rate of other relaxation channels (e.g., optical phonon emission), we expect these channels to start contributing to the overall energy relaxation. This would lead to a decrease in the fraction of energy that is transferred to the electron system, that is, a reduced carrier heating efficiency.

We quantify the fraction of absorbed energy that leads to carrier heating by comparing our $\mathrm{THz}$ photoconductivity data with the results of a simple thermodynamic model. In short, carrier heating leads to a broader carrier distribution (higher $T_{\mathrm{el}}$ ), which in combination with an energy-dependent scattering time $^{25,26}$ leads to the photoinduced change in $\mathrm{THz}$ conductivity (see Methods). The carrier heating is governed by the amount of absorbed energy $F_{\mathrm{abs}}$ and the electronic heat capacity of graphene, which for a degenerate electron gas is given by $C_{\mathrm{el}}=$ $\alpha T_{\mathrm{el} .}{ }^{27}$ Here $\alpha=\left[\left(2 \pi E_{\mathrm{F}}\right) /\left(3 \hbar^{2} v_{\mathrm{F}}^{2}\right)\right] k_{\mathrm{B}}^{2}$, with $\hbar, v_{\mathrm{F}}$ and $k_{\mathrm{B}}$ the reduced Planck constant, the Fermi velocity, and Boltzmann's constant, respectively. The possibility of controlling the Fermi energy of graphene thus allows for tunability of the heat capacity (see Figure 2a), which in turn determines the "hot" carrier temperature $T_{\mathrm{el}}^{\prime}$ that the system reaches. Figure $2 \mathrm{~b}$
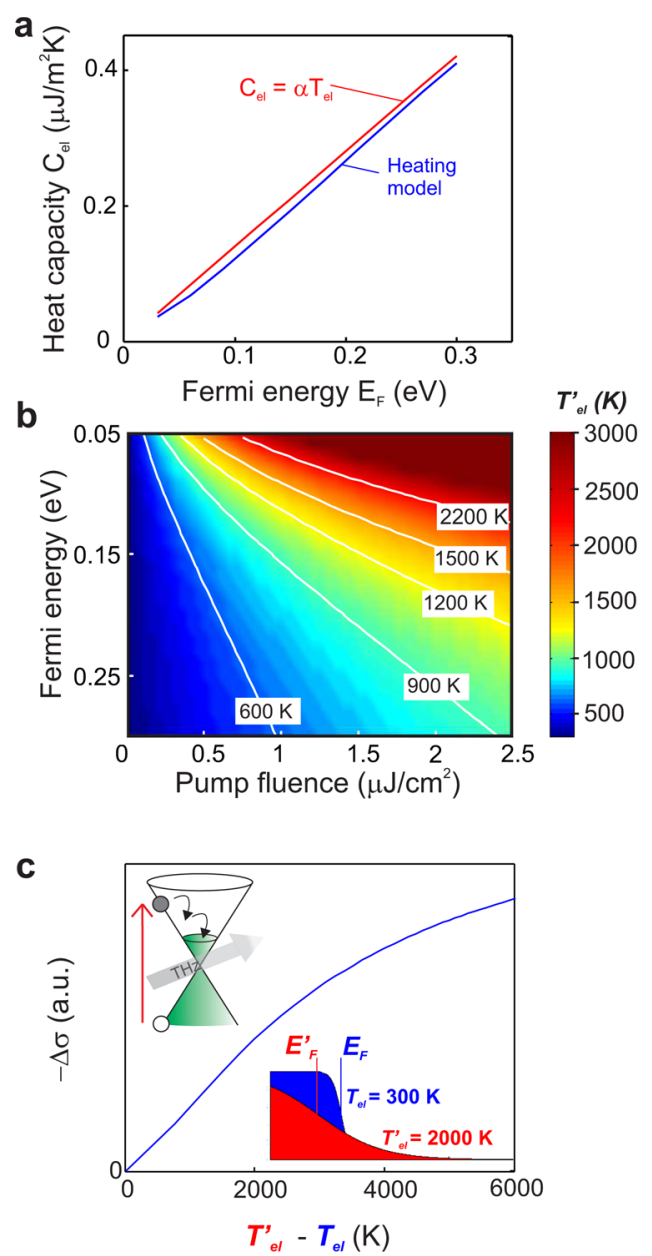

Figure 2. Carrier heating model. (a) The electronic heat capacity according to the analytical theory (red line) and our numerical model (blue line), showing linear scaling with Fermi energy. (b) Contour plot of the temperature of the carrier population, showing that decreasing the Fermi energy and increasing the fluence have an equivalent effect on the temperature of the electrons after photoexcitation and intraband thermalization. The carrier temperature is calculated through a simple thermodynamic model (see text). (c) The calculated negative $\mathrm{THz}$ photoconductivity as a function of carrier temperature, showing a linear dependence up to $\sim 2000 \mathrm{~K}$. The top left inset shows schematically the process of ultrafast energy relaxation of a photoexcited carrier, which leads to an increase of the carrier temperature from $T_{\mathrm{el}}$ to $T_{\mathrm{el}}^{\prime}$. The inset on the bottom right shows the Fermi-Dirac distribution of the conduction band carriers, where the temperature increase leads to a decrease of the Fermi energy (to keep the total number of conduction band carriers constant). 
shows that $T_{\mathrm{el}}^{\prime}$ is equivalently determined by both the fluence and the Fermi energy.

To calculate the hot carrier temperature we use a basic numerical approach, which produces a heat capacity in the degenerate regime that corresponds well with the analytical heat capacity (see Figure 2a), while remaining valid for nondegenerate electron temperatures $\left(k_{\mathrm{B}} T_{\mathrm{e} l}>E_{\mathrm{F}}\right)$. The numerical approach is based on the concept that before photoexcitation there is a known amount of energy in the electronic system, $\varepsilon_{1}=\int_{0}^{\infty} \mathrm{d} \varepsilon D(\varepsilon) \varepsilon F\left(E_{\mathrm{F}}, T_{\mathrm{el}}\right)$, and a known number of carriers in the conduction band, $N_{\text {int }}=\int_{0}^{\infty} \mathrm{d} \varepsilon D(\varepsilon)$ $F\left(E_{\mathrm{F}}, T_{\mathrm{el}}\right)$, where $D(\varepsilon)$ is the energy-dependent density of states and $F\left(E_{\mathrm{F}}, T_{\mathrm{el}}\right)$ is the Fermi-Dirac distribution that depends on Fermi energy $E_{\mathrm{F}}$ and carrier temperature $T_{\mathrm{el}}$. Because of optical excitation, an amount of energy $F_{\text {abs }}$ is absorbed in the graphene and a fraction $\eta$ of this energy ends up in the electronic system trough intraband carrier-carrier scattering. After intraband heating is complete, the system is then described by the following set of equations: $\mathcal{E}_{2}=\mathcal{E}_{1}+$ $\eta F_{\mathrm{abs}}=\int_{0}^{\infty} \mathrm{d} \varepsilon D(\varepsilon) \varepsilon F\left(E_{\mathrm{F}}^{\prime} T_{\mathrm{el}}^{\prime}\right)$, and (conserving the number of conduction band carriers) $N_{\text {int }}=\int_{0}^{\infty} \mathrm{d} \varepsilon D(\varepsilon) F\left(E_{\mathrm{F}}^{\prime} T_{\mathrm{el}}^{\prime}\right)$. Here $E_{\mathrm{F}}^{\prime}$ and $T_{\mathrm{el}}^{\prime}$ are the chemical potential and the carrier temperature in the hot state, respectively. Carrier heating thus alters the carrier distribution, where we find by numerically solving the equations for $\mathscr{E}_{2}$ and $N_{\text {int }}$ that the carrier temperature increases and the chemical potential decreases upon photoexcitation (see inset of Figure $2 \mathrm{c}$ and Methods). The photoinduced increase of carrier temperature and the associated decrease in chemical potential were experimentally confirmed recently. ${ }^{13}$

The hot carrier distribution, with $E_{\mathrm{F}}^{\prime}$ and $T_{\mathrm{el}}^{\prime}$ calculated using the carrier heating model, directly leads to negative $\mathrm{THz}$ photoconductivity (see Methods), which scales linearly with carrier temperature up to $\sim 2000 \mathrm{~K}$ and then shows some saturation behavior (Figure 2c). We test the validity of our carrier heating model by comparing its predictions for the frequency-resolved photoconductivity with our experimental results for the sample with fixed Fermi energy. In Figure 3a, we show this comparison for a fluence of $\sim 12 \mu \mathrm{J} / \mathrm{cm}^{2}$ (pump wavelength $800 \mathrm{~nm}, N_{\text {exc }}=1 \times 10^{12}$ absorbed photons $/ \mathrm{cm}^{2}$ ). We compare the data (at the time delay that corresponds to the signal peak) with the model result for a ground state Fermi energy of $0.11 \mathrm{eV}$ and a scattering time proportionality constant of $200 \mathrm{fs} / \mathrm{eV}$ (extracted from the Raman spectrum, $\mathrm{THz}$ conductivity measurements on the same sample without photoexcitation, and the saturation value of the $\mathrm{THz}$ photoconductivity at high fluence; see also Supporting Information) and find good agreement with a heating efficiency of $\eta=0.75$. The small discrepancies between data and model can be ascribed to artifacts that arise from the temporal change of the photoconductivity during the interaction with the $\mathrm{THz}$ pulse, ${ }^{28}$ although we largely avoid these by moving the optical pump delay line simultaneously with the $\mathrm{THz}$ probe delay line.

The overall agreement between data and model shows that the observed negative $\mathrm{THz}$ photoconductivity of intrinsically doped graphene $e^{11,16,17,20-22}$ can be fully reproduced by considering intraband carrier heating, which reduces the thermally averaged conductivity of the intrinsic carriers. Despite the simplicity of the model, it can also explain the experimental results in ref 21 using their experimental parameters, as well as the results in ref 20 , by letting the environmental gas change the Fermi energy. These observations lead us to conclude that, despite some uncertainty in the determination of the Fermi energy and the scattering time, the model is suitable for
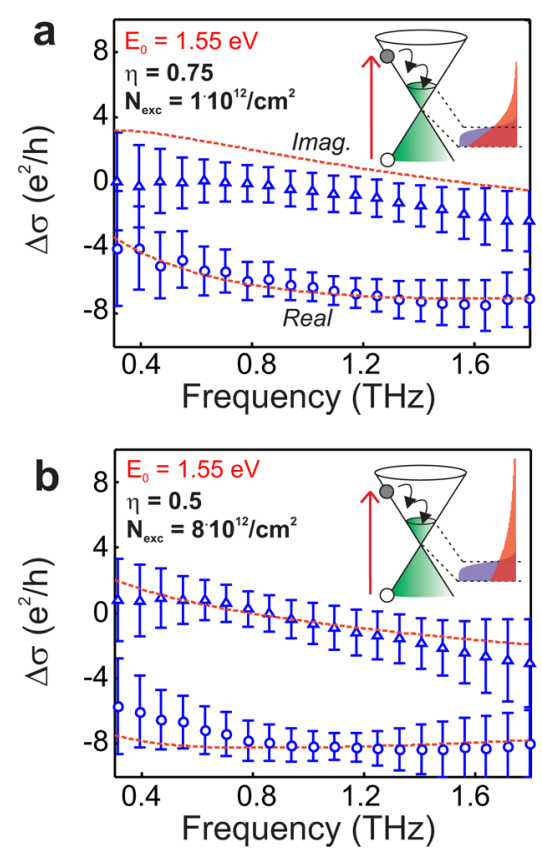

Figure 3. Comparison of experimental and theoretical photoconductivity. (a) The complex photoconductivity of the sample with fixed Fermi energy as a function of frequency for an excitation fluence corresponding to $N_{\mathrm{exc}}=1 \times 10^{12}$ carriers $/ \mathrm{cm}^{2}$, together with the model result with a carrier heating efficiency of $\eta=0.75$. The model (see also main text and Methods) describes the photoconductivity after photoexcited carriers have thermalized through intraband carrier-carrier scattering and directly yields the observed negative photoconductivity. (b) The complex photoconductivity of the sample with fixed Fermi energy as a function of frequency for an excitation fluence corresponding to $N_{\text {exc }}=8 \times 10^{12}$ carriers $/ \mathrm{cm}^{2}$, together with the model result with a carrier heating efficiency of $\eta=0.5$. This shows that in this regime other energy relaxation channels contribute to the ultrafast energy relaxation. The insets in panels $a$ and $b$ schematically show the process of carrier heating through intraband scattering and the corresponding carrier distributions for the ambient carrier temperature $T_{\mathrm{el}}$ (blue) and the elevated carrier temperature $T_{\mathrm{el}}^{\prime}$ after photoexcitation and carrier thermalization (red). The error bars in panels $\mathrm{a}$ and $\mathrm{b}$ represent the experimental (nonsystematic) error bars ( $95 \%$ confidence interval).

obtaining reliable qualitative indications on how the carrier heating efficiency depends on the Fermi energy and the fluence.

We now examine the "high fluence" regime using $N_{\text {exc }}=8 \times$ $10^{12}$ absorbed photons $/ \mathrm{cm}^{2}$ (a fluence of $\sim 100 \mu \mathrm{J} / \mathrm{cm}^{2}$ ) in Figure $3 \mathrm{~b}$. This corresponds to the regime where the energy relaxation is slower than the experimental time resolution (see Figure 1d). Here we find that we can only describe the data with a significantly reduced carrier heating efficiency of $\eta \approx 0.5$ (keeping the ground state Fermi energy and scattering time proportionality constant the same as in the low fluence regime). Combined, these results show that at sufficiently low fluence, a large fraction of the absorbed energy ends up in the electron system, that is, the ultrafast energy relaxation occurs through efficient (and fast) carrier-carrier scattering. However, upon increasing the fluence (i.e., the carrier temperature), the relative amount of energy transferred to the electron system decreases, which means that carrier-carrier scattering becomes less efficient (and slower) and other relaxation processes start to contribute.

To determine in more detail how the carrier heating efficiency depends on fluence, we study the peak photo- 
conductivity of the sample with fixed Fermi energy for a large range of excitation powers for both 800 and $400 \mathrm{~nm}$ excitation. We show the photoconductivity at the peak (when the ultrafast energy relaxation is complete) in Figure $4 a, b$, together with the

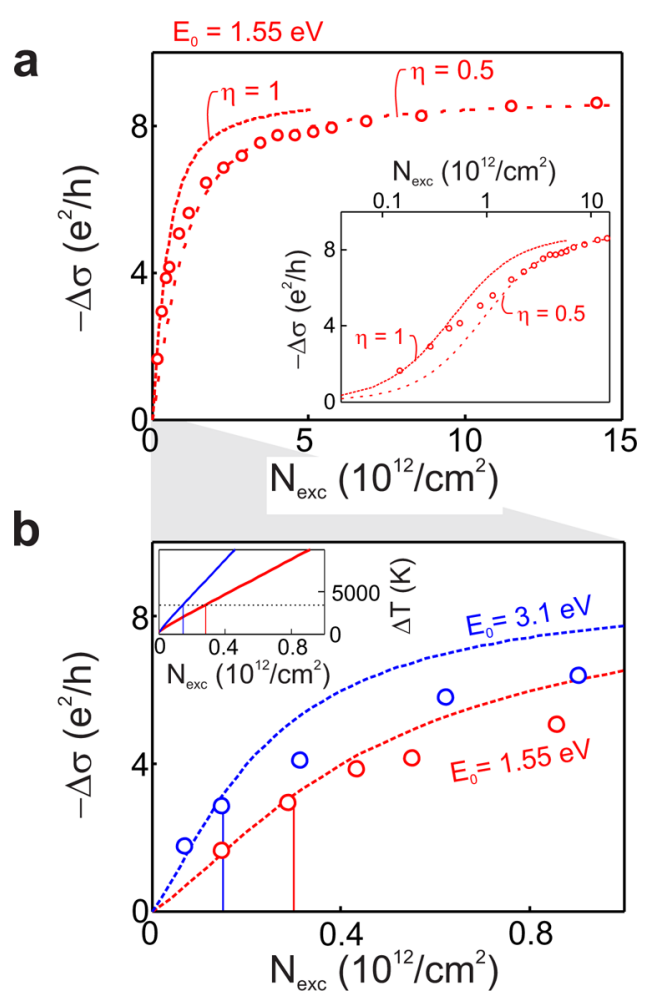

Figure 4. Carrier heating efficiency-dependence on fluence. (a) The peak photoconductivity of the sample with fixed Fermi energy for a large range of excitation powers and an excitation wavelength of 800 $\mathrm{nm}$ together with the model with $\eta=1$ (short dashed line) and a heating efficiency of $\eta=0.5$ (long dashed line). The inset shows the same data on a logarithmic horizontal scale. (b) The peak photoconductivity of the sample with fixed Fermi energy as a function of excitation power $\left(N_{\text {exc }}\right)$ for excitation with $800 \mathrm{~nm}$ light (corresponding to an energy of the primary excited carrier of $E_{0} / 2$ $=0.75 \mathrm{eV})$ and $400 \mathrm{~nm}$ light $\left(E_{0} / 2=1.55 \mathrm{eV}\right)$. The dashed lines correspond to the model with the same parameters (Fermi energy and scattering time proportionality constant) as in panel a, using $\eta=1$. The model describes the data well up to $N_{\text {exc }}=0.3 \times 10^{12}$ carriers $/ \mathrm{cm}^{2}$ for $800 \mathrm{~nm}$ excitation and up to $N_{\text {exc }}=0.15 \times 10^{12}$ carriers $/ \mathrm{cm}^{2}$ for $400 \mathrm{~nm}$ excitation. The inset shows the carrier temperature that is reached after excitation and thermalization by carrier-carrier scattering, reaching $\sim 4000 \mathrm{~K}$ before heating becomes less efficient, for both excitation wavelengths. The symbol size of the data in panels $\mathrm{a}$ and $\mathrm{b}$ represents the error.

results of the carrier heating model for the same parameters as in Figure $3 \mathrm{a}$ and a frequency of $0.7 \mathrm{THz}$. For low fluences (up to $\sim 4 \mu \mathrm{J} / \mathrm{cm}^{2}$, corresponding to $N_{\text {exc }} \sim 0.3 \times 10^{12}$ absorbed photons $/ \mathrm{cm}^{2}, 800 \mathrm{~nm}$ excitation, $\sim 2 \%$ absorption) the experimental data are in agreement with the heating model with a fixed heating efficiency of $\eta=1$. We notice that the calculated photoconductivity shows saturation behavior with $N_{\text {exc }}$ even for constant $\eta$. This is related to the nonlinear dependence of the $\mathrm{THz}$ photoconductivity on carrier temperature (Figure 2c). Interestingly, at fluences above $\sim 4 \mu \mathrm{J} / \mathrm{cm}^{2}$ the experimental photoconductivity starts saturating and the model is only in agreement for a heating efficiency that gradually decreases to $\sim 50 \%$ for the highest fluences applied here (Figure 4a). These observations suggest that once a certain carrier temperature $(\sim 4000 \mathrm{~K}$, see inset Figure $4 \mathrm{~b})$ is reached, the heating efficiency decreases. Interestingly, the experimental data for excitation with $400 \mathrm{~nm}$ light start deviating from the model (with efficient heating) at $N_{\text {exc }} \sim 0.15$ $\times 10^{12}$ absorbed photons $/ \mathrm{cm}^{2}$ (Figure $4 \mathrm{~b}$ ), instead of $\sim 0.3 \times$ $10^{12}$ absorbed photons $/ \mathrm{cm}^{2}$ in the case of excitation with 800 $\mathrm{nm}$ light. This is because each $400 \mathrm{~nm}$ photon has twice the energy of a $800 \mathrm{~nm}$ photon. Thus, in both cases the carrier heating efficiency starts decreasing around the same carrier temperature.

We now determine how the heating efficiency depends on the Fermi energy by measuring the peak photoconductivity for the sample with controllable Fermi energy as a function of both excitation power and Fermi energy. The combined results (for excitation at $1500 \mathrm{~nm}$ ) are represented in Figure 5a, where we



show the peak photoconductivity as a function of gate voltage for five different excitation powers. In Figure 5b, we show the peak photoconductivity as a function of $N_{\text {exc }}$ for three distinct gate voltages, corresponding to $N_{\text {int }} \approx 1,2$, and $3 \times 10^{12}$ carriers $/ \mathrm{cm}^{2}$, and compare these to the results of the heating model. We find that for a doping of $N_{\text {int }}=3 \times 10^{12}$ carriers $/ \mathrm{cm}^{2}$ $\left(E_{\mathrm{F}} \sim 0.2 \mathrm{eV}\right)$ the data is in good agreement with the carrier heating model, using a scattering time proportionality constant 
of $\sim 50 \mathrm{fs} / \mathrm{eV}$ and a carrier heating efficiency of $\eta=1$. However, for the lowest $N_{\text {int }}$, which corresponds to a Fermi energy of $\sim 0.1 \mathrm{eV}$, we find a carrier heating efficiency of $\sim 20 \%$, using the same energy-dependent scattering time. These results are in good agreement with the observed slowdown of the rise dynamics with decreasing Fermi energy in Figure $1 b$.

Comparing the data and the heating model leads to the following physical picture of the ultrafast energy relaxation in graphene: Until a certain carrier temperature is reached $(\sim 4000$ $\mathrm{K})$, the ultrafast energy relaxation is dominated by carriercarrier scattering, which leads to efficient and fast $(<150 \mathrm{fs})$ carrier heating. Once this carrier temperature is reached, the relaxation slows down and the carrier heating efficiency decreases, as ultrafast energy relaxation occurs through additional pathways involving optical phonon emission. ${ }^{14}$ The reduction in heating efficiency that follows from the macroscopic heating model can be explained using the microscopic picture of intraband carrier-carrier scattering, as put forward in refs 10 and 11. At increased electron temperatures, the (quasiequilibrium) Fermi energy decreases (see Methods), which means that the electronic heat capacity decreases. It furthermore implies that the amount of energy that is exchanged in intraband carrier-carrier scattering events $\left(\sim E_{\mathrm{F}}\right)$ decreases. Therefore, energy relaxation of a photoexcited carrier requires an increasing number of intraband carriercarrier scattering cascade steps. Thus, for an increasing carrier temperature, energy relaxation through intraband carrier heating slows down.

The physical picture of carrier-temperature dependent ultrafast energy relaxation of photoexcited carriers in graphene unites the conclusions of a large fraction of the existing literature on this topic. For example, in ref 14 with excitation in the "high fluence" regime $\left(\sim 10^{14}\right.$ absorbed photons $/ \mathrm{cm}^{2}$ at 800 $\mathrm{nm}$ ) it was concluded from the experimentally measured carrier temperature that only part of the absorbed light energy ends up in the electronic system, whereas the rest couples to optical phonons. An ultrafast optical pump-probe study employing a fluence of $200 \mu \mathrm{J} / \mathrm{cm}^{2}$, also in the high fluence regime, similarly demonstrated optical phonon mediated relaxation, in addition to carrier heating. By measuring in the "low fluence" regime (a few microjoules), ref 11 concluded that the ultrafast energy relaxation was dominated by carrier heating. Furthermore, two very recent optical pump-THz probe studies ${ }^{16,17}$ both used an excited carrier density of $N_{\mathrm{exc}} \approx 1 \times 10^{12}$ absorbed photon $/ \mathrm{cm}^{2}$ at $800 \mathrm{~nm}$ and ascribe their observed negative $\mathrm{THz}$ photoconductivity (partially) to carrier heating.

In conclusion, we provide a unifying explanation of the ultrafast energy relaxation of photoexcited carriers in graphene. For sufficiently low excitation power and sufficiently high Fermi energy, the relaxation is dominated by carrier-carrier scattering, which leads to efficient generation of hot carriers. This regime typically persists up to a fluence of $\sim 4 \mu \mathrm{J} / \mathrm{cm}^{2}$ (or $N_{\text {exc }}=0.3 \times 10^{12}$ absorbed $800 \mathrm{~nm}$ photons $/ \mathrm{cm}^{2}$ ) for a Fermi energy of $0.1 \mathrm{eV}$. For larger Fermi energy, a higher fluence can be used without significant reduction in heating efficiency. In the case of lower Fermi energy and/or a higher fluence, the heating efficiency will decrease due to slower intraband carriercarrier scattering and additional energy relaxation channels involving optical phonon emission. This opens up the possibility to control the pathway of ultrafast energy relaxation, that is, the ability to tune the efficiency of energy transfer from the primary excited carriers to electronic heat or to alternative degrees of freedom, such as lattice heat. Such tunability is useful for future applications, for instance in the field of photodetection, where hot carriers are the dominant source of photocurrent generation. ${ }^{6}$ Finally, we note that the terrestrial solar radiation (on the order of a $\mathrm{pJ} / \mathrm{cm}^{2}$ during a $10 \mathrm{ps}$ time scale) corresponds to the low fluence regime with efficient carrier heating, which is therefore the relevant process to consider for photovoltaic applications.

Methods. Photoconductivity from Carrier Heating Model. We use a numerical model based on carrier heating to calculate the complex photoconductivity of photoexcited graphene. The frequency-dependent conductivity of graphene is generally given by $^{26}$

$$
\sigma(\omega)=\frac{e^{2} v_{\mathrm{F}}^{2}}{2} \int_{0}^{\infty} \mathrm{d} \varepsilon D(\varepsilon) \frac{\tau_{\text {scatter }}(\varepsilon)}{1-i \omega \tau_{\text {scatter }}(\varepsilon)} \frac{\mathrm{d} F\left(E_{\mathrm{F}}, T_{\mathrm{el}}\right)}{\mathrm{d} \varepsilon}
$$

with $e$ as the elementary charge, $\nu_{\mathrm{F}}$ is the Fermi velocity, $D(\varepsilon)=$ $2 \varepsilon /\left(\pi \hbar^{2} \nu_{\mathrm{F}}^{2}\right)$ is the density of states, $\hbar$ is the reduced Planck constant, $\tau_{\text {scatter }}(\varepsilon)$ is the energy-dependent momentum scattering time, and $F\left(E_{\mathrm{F}}, T_{\mathrm{el}}\right)$ is the Fermi-Dirac distribution that is determined by the Fermi level and the carrier temperature. For unexcited graphene we use the steady state Fermi level $E_{\mathrm{F}}$ and the ambient temperature $T_{\mathrm{el}}$. We furthermore use a scattering time that is determined by charged impurity scattering and increases linearly with energy $\varepsilon^{25,26}$ After electron-hole pair excitation of graphene, photoexcited carriers can interact with the intrinsic carriers, leading to intraband thermalization (see also main text); the carrier temperature increases to $T_{\mathrm{el}}^{\prime}$ and the chemical potential decreases to $E_{\mathrm{F}}^{\prime}$. The reason for the decrease of the chemical potential is the linear scaling of the $D(\varepsilon)$ with energy; a broader carrier distribution (higher carrier temperature) would lead to an increased number of carriers in the conduction band if the Fermi energy would be kept constant. Therefore, a higher carrier temperature leads to a lower Fermi energy, as confirmed experimentally in ref 13 . The conductivity of photoexcited graphene $\sigma^{\prime}(\omega)$ then follows from eq 1, using the "hot Fermi level" and "hot carrier temperature" with the photoconductivity given by $\Delta \sigma(\omega)=\sigma^{\prime}(\omega)-\sigma(\omega)$.

Our model takes into account the effect of intraband carrier heating on the photoconductivity through the carrier distribution, which is sufficient to explain the observed negative photoconductivity ${ }^{11,16,17,20-22}$ and the dependence on excitation power and Fermi energy. The model does not explicitly include the effect of energy relaxation to optical phonons on the photoconductivity, as proposed in ref 22 . This is justified, because for the graphene used here (with an impurityscattering-limited mobility below $2500 \mathrm{~cm}^{2} /(\mathrm{V} \mathrm{s})$ ) the effect of phonons on the conductivity is negligibly small at low fluences. ${ }^{11}$ We note that for very high fluences $\left(N_{\text {exc }}>10^{12}\right.$ absorbed photons $/ \mathrm{cm}^{2}$ ) and for phonon-scattering-limited graphene with mobilities $>10,000 \mathrm{~cm}^{2} /(\mathrm{V} \mathrm{s}),{ }^{29}$ this effect likely plays a role, in addition to carrier heating. Furthermore, a more advanced model could include deviations from linear scaling between the scattering time and the carrier energy, ${ }^{30}$ as well as changes in the Drude weight. The latter effect occurs when the valence and conduction band electrons no longer have separate thermal distributions and likely plays an important role around the Dirac point, where it correctly produces positive photoconductivity. ${ }^{17}$

Rise Dynamics. We describe the time-resolved photoconductity with a phenomenological model that includes a 
rise step and an exponential decay step with time $\tau_{\text {decay }}$. In the low excitation power/high Fermi energy regime (highly efficient carrier heating), the rise occurs within our instrument response function. For high excitation power/low Fermi energy, the rise contains a very fast component and a slow component (reduced carrier heating efficiency). Therefore, we model the rise dynamics with a "fast" rise component that is fixed at a value equal to the pulse duration of $\tau_{\text {pulse }}=120-150$ fs (depending on the excitation wavelength) and a variable "slow" rise component $\tau_{\text {slow }}$ with a longer pulse duration and describe the time-resolved photoconductivity by

$$
\begin{aligned}
-\Delta \sigma(t)= & A \cdot \operatorname{Conv}\left(\mathrm{e}^{-t / \tau_{\text {decay }}}, \tau_{\text {pulse }}\right) \\
& +B \cdot \operatorname{Conv}\left(\mathrm{e}^{-t / \tau_{\text {decay }}}, \tau_{\text {slow }}\right)
\end{aligned}
$$

where $A$ and $B$ are the amplitudes corresponding to the fast and the slow rise components and $\operatorname{Conv}(y(t), \tau)$ means taking the convolution of $y(t)$ with a Gaussian pulse of width $\tau$. The effective rise time is then given by

$$
\tau_{\text {rise }}=\frac{A+B}{\frac{A}{\tau_{\text {pulse }}}+\frac{B}{\tau_{\text {slow }}}}
$$

In the fit, the free parameters are $A, B, \tau_{\text {slow, }}$ and $\tau_{\text {decay. }}$. The resulting effective rise time gives an indication of how fast the initial energy relaxation of photoexcited carriers takes place.

\section{ASSOCIATED CONTENT}

\section{S Supporting Information}

Supporting Information Available: Sample characterization and additional measurements. This material is available free of charge via the Internet at http://pubs.acs.org.

\section{AUTHOR INFORMATION}

\section{Corresponding Authors}

*E-mail: (F.H.L.K.) frank.koppens@icfo.es.

*E-mail: (M.B.) bonn@mpip-mainz.mpg.de.

*E-mail: (K.J.T.) klaas-jan.tielrooij@icfo.es.

\section{Notes}

The authors declare no competing financial interest.

\section{ACKNOWLEDGMENTS}

We would like to thank Justin Song, Leonid Levitov, Sebastien Nanot, and Enrique Cánovas for useful discussions. K.J.T. thanks NWO for a Rubicon fellowship. F.K. acknowledges support by the Fundacio Cellex Barcelona, the ERC Career integration Grant 294056 (GRANOP), the ERC starting grant 307806 (CarbonLight) and support by the E. C. under Graphene Flagship (contract no. CNECT-ICT-604391).

\section{REFERENCES}

(1) Bonaccorso.; et al. Graphene Photonics and Optoelectronics. Nat. Photonics 2010, 4, 611.

(2) Dawlaty, J. M.; et al. Measurement of the optical absorption spectra of epitaxial graphene from terahertz to visible. Appl. Phys. Lett. 2008, 93, 131905.

(3) Ren, L.; et al. Terahertz and Infrared Spectroscopy of Gated Large-Area Graphene. Nano Lett. 2012, 12, 3711-3715.

(4) Novoselov, K. S.; et al. Electric field effect in atomically thin carbon films. Science 2004, 306, 666-669.

(5) Wang, L.; et al. One-Dimensional Electrical Contact to a TwoDimensional Material. Science 2013, 342, 614-617.

(6) Gabor, N. M.; et al. Hot Carrier-Assisted Intrinsic Photoresponse in Graphene. Science 2011, 334, 648-652.
(7) Winzer, T.; Knorr, A.; Malić, E. Carrier Multiplication in Graphene. Nano Lett. 2010, 10, 4839-4843.

(8) Brida, D.; et al. Ultrafast collinear scattering and carrier multiplication in graphene. Nat. Commun. 2013, 4, 1987.

(9) Plötzing, T. Nano Lett. 2014, DOI: 10.1021/nl502114w.

(10) Song, J. C. W.; et al. Photoexcited carrier dynamics and impactexcitation cascade in graphene. Phys. Rev. B 2013, 87, 155429.

(11) Tielrooij, K. J.; et al. Photoexcitation cascade and multiple hotcarrier generation in graphene. Nat. Phys. 2013, 9, 248-252.

(12) Johannsen, J. C.; et al. Direct view on the ultrafast carrier dynamics in graphene. Phys. Rev. Lett. 2013, 11, 027403.

(13) Gierz, I.; et al. Snapshots of non-equilibrium Dirac carrier distributions in graphene. Nat. Mater. 2013, 12, 1119-1124.

(14) Lui, C. H.; et al. Ultrafast photoluminscence from graphene. Phys. Rev. Lett. 2010, 105, 127404.

(15) Breusing, M.; et al. Ultrafast nonequilibrium carrier dynamics in a single graphene layer. Phys. Rev. B 2011, 83, 153410.

(16) Shi, S.-F.; et al. Controlling Graphene Ultrafast Hot Carrier Response from Metal-like to Semiconductor-like by Electrostatic Gating. Nano Lett. 2014, 14, 1578-1582.

(17) Frenzel, A. J.; et al. Semiconducting-to-metallic photoconductivity crossover and temperature-dependent Drude weight in graphene. Phys. Rev. Lett. 2014, 113, 056602.

(18) Strait, J. H.; et al. Very Slow Cooling Dynamics of Photoexcited Carriers in Graphene Observed by Optical-Pump Terahertz-Probe Spectroscopy. Nano Lett. 2011, 11, 4902-4906.

(19) George, P. A.; et al. Ultrafast Optical-Pump Terahertz-Probe Spectroscopy of the Carrier Relaxation and Recombination Dynamics in Epitaxial Graphene. Nano Lett. 2008, 8, 4248-4251.

(20) Docherty, C. J.; et al. Extreme sensitivity of graphene photoconductivity to environmental gases. Nat. Commun. 2012, 3, 1228.

(21) Frenzel, A. J.; et al. Observation of suppressed terahertz absorption in photoexcited graphene. Appl. Phys. Lett. 2013, 102, 113111.

(22) Jnawali, G.; et al. Observation of a Transient Decrease in Terahertz Conductivity of Single-Layer Graphene Induced by Ultrafast Optical Excitation. Nano Lett. 2013, 13, 524-530.

(23) Ulbricht, R; et al. Carrier dynamics in semiconductors studied with time-resolved terahertz spectroscopy. Rev. Mod. Phys. 2011, 83, 543.

(24) Pijpers, J. J. H.; et al. Assessment of carrier-multiplication efficiency in bulk PbSe and PbS. Nat. Phys. 2009, 5, 811.

(25) Ando, T. Screening Effect and Impurity Scattering in Monolayer Graphene. J. Phys. Soc. Jpn. 2006, 75, 074716.

(26) Das Sarma, S.; Adam, S.; Hwang, E. H.; Rossi, E. Electronic transport in two-dimensional graphene. Rev. Mod. Phys. 2011, 83, 407.

(27) Kittel, C. Introduction to Solid State Physics; Wiley and Sons: New York, 2005.

(28) Hendry, E.; et al. Interchain effects in the ultrafast photophysics of a semiconducting polymer: $\mathrm{THz}$ time-domain spectroscopy of thin films and isolated chains in solution. Phys. Rev. B 2005, 71, 125201.

(29) Perebeinos, V.; Avouris, P. Inelastic scattering and current saturation in graphene. Phys. Rev. B 2010, 81, 195442.

(30) Hwang, E. H.; Adam, S.; Das Sarma, S. Carrier Transport in Two-Dimensional Graphene Layers. Phys. Rev. Lett. 2007, 98, 186806. 